\title{
Proteinase-activated receptors $I$ and 2 activate protein kinase $D I$ in human melanoma cells
}

\author{
C Kempkes*1, A Rattenholl11, GS Bocheva², KS Hoek³ , A Haußer, \\ R Dummer ${ }^{3}$ and M Steinhoff 1
}

Address: ${ }^{1}$ University of Münster, Department of Dermatology, Münster, Germany, ${ }^{2}$ Medical University of Sofia, Department of Dermatology and Venereology, Sofia, Bulgaria, ${ }^{3}$ University Hospital of Zürich, Department of Dermatology, Zürich, Switzerland and ${ }^{4}$ University of Stuttgart, Institute of Cell Biology and Immunology, Stuttgart, Germany

* Corresponding author

from 12th Joint Meeting of the Signal Transduction Society (STS). Signal Transduction: Receptors, Mediators and Genes Weimar, Germany. 29-31 October 2008

Published: 26 February 2009

Cell Communication and Signaling 2009, 7(Suppl I):A99 doi:10.1 I86/I478-8IIX-7-SI-A99

This abstract is available from: http://www.biosignaling.com/content/7/SI/A99

(c) 2009 Kempkes et al; licensee BioMed Central Ltd.

Proteinase-activated receptors (PARs) are G-protein coupled receptors with seven transmembrane domains that are stimulated by a unique activation mechanism. The extracellular N-terminus is specifically cleaved by certain serine proteinases, which exposes a formerly latent peptide sequence. This "tethered ligand" is able to bind to the second extracellular loop of the receptor, leading to the transduction of signal events. Four PARs have been cloned so far. $\mathrm{PAR}_{1}, \mathrm{PAR}_{3}$ and $\mathrm{PAR}_{4}$ are stimulated by thrombin, whereas $\mathrm{PAR}_{2}$ can be activated by trypsin. In vitro, PARs can also be stimulated by synthetic peptides, which mimic the tethered ligand sequence. $\mathrm{PAR}_{1}$, but not $\mathrm{PAR}_{2}$, is expressed by primary melanocytes. It is well known that $\mathrm{PAR}_{1}$ is overexpressed in malignant melanomas, enhancing migration and metastasis. $\mathrm{PAR}_{2}$ also seems to play a role in melanoma progression and metastasis. The underlying mechanisms, however, still remain elusive. Stimulation of both receptors on the the human melanoma cell line WM9 led to rearrangement of integrin $\alpha v \beta 3$ and phosphorylation of protein kinase D1 (PKD1). PKD1 is known to control persistent cell migration by regulating the intracellular integrin $\alpha v \beta 3$ recycling pathway. Stable knockdown of PKD1 in WM9 cells led to an inhibition of cell proliferation, changes in cell shape and a downregulation of integrin $\alpha v \beta 3$ on the cell surface. Moreover, cell migration was impaired. Taken together, we could show for the first time that both $\mathrm{PAR}_{1}$ and $\mathrm{PAR}_{2}$ are involved in melanoma cell migration and proliferation via activation of PKD1. 